\title{
SPIN-COATING OF DILUTE MAGNETIC COLLOIDS IN A MAGNETIC FIELD
}

\author{
M. Pichumani, W. González-Viñas \\ Department of Physics and Applied Mathematics, University of Navarra, \\ Irunlarrea s/n, 31080 Pamplona, Spain
}

\begin{abstract}
Spin-coating of colloids is a versatile method in fabricating films of colloidal particles at a short span of time. Controlling key parameters like the rate of rotation, the initial concentration and the nature of the continuum phase is easy to perform experimentally. However, the effects of these parameters are not fully understood yet. To enhance the understanding of the coating process, we study the spin-coating of dilute magnetic colloids. The coating process is controlled externally by means of a magnetic field and we investigate its effects by studying the morphology of the dried coating obtained after the experiments. Morphological transitions are explained through the mean area and the number density of clusters, together with anisotropy properties. Further, we relate the occupation factor of clusters to the non-planarization conditions observed in this kind of experiments.
\end{abstract}

Introduction. Dispersions of magnetic particles significantly change their behaviour when introduced in a homogeneous magnetic field. The magnetic particles form clusters, which change the overall properties of the dispersion under stresses. In other words, an applied magnetic field can change the rheological properties of magnetic colloids. In this framework, we pour a sample of the dispersion onto a rapidly rotating substrate. We investigate the effects due to the applied field and compare them to the case of zero-field condition. If the continuum phase of the dispersion is a volatile solvent, it evaporates as the rotation continues and leaves a solid coating of colloidal magnetic particles. This process is done with the help of a commercial spin-coater and a Helmholtz pair.

The fabrication of magnetic thin film devices is gaining research and commercial interests $[1,2]$. By varying the size of the magnetic particles, their applications can be classified. Among other ways, self assembly of magnetic particles is of particular interest due to lower complexity during the fabrication and comparatively high quality structures [3, 4]. Spin-coating of colloidal dispersion has a great advantage in producing resultant morphologies at a short span of time [5-7].

Although cluster formation in magnetic colloids has been investigated numerically [8-15], the kind of colloidal particles (nano-sized) and the high concentration (typical of ferrofluids) have undergone difficult experimental studies. Lately some groups paid attention to core-shell superparamagnetic particles (micron-sized) at low dilution, much easier to characterize in experiments under constant magnetic fields [16], or under rotating magnetic fields ([17], among others). The main difference of these works from our system is the presence of inertial effects in the fluid, such as a centrifugal force, and the existence of free surface, where the solvent is evaporating.

Spin-coating of simple fluids is well known, and there are models [18, 19] based on the lubrication approximation, which predict fairly well the experimental results [20]. Concerning the spin-coating of colloids, the first studies (both numerical simulations and experiments) [21] focused on the effect of some param- 


\section{Pichumani, W. González-Viñas}

eters on the thickness of the coating. Later on, non-planar coatings were obtained in experiments [7], and symmetry transitions, which resulted from shear melting and shear crystallization mechanisms [22, 23], were studied. Many questions concerning these systems still remain open and it is worth exploring the physics behind these phenomena.

By controlling the spinning rate and the initial concentration of the dispersion, the kind of final morphologies can be tuned. Previous experiments were done at medium or high concentrations, which made the characterization of the clustering processes more difficult. The clustering processes are very relevant to the final deposit and its order. In this work, we will focus on the spin-coating of dilute magnetic colloids. An external axial magnetic field affects the rheology of the suspension, as well as it could vary the aggregation dynamics. This could shed light on the transition from sub-monolayer to compact structures.

1. Experimental set-up. The experiments are performed in a spin-coater working at rotation rates from 2000 to $6000 \mathrm{rpm}$. Helmholtz coils are placed in such a way that the substrates can spin in the region of uniform axial magnetic field (Fig. 1). The applied magnetic fields are varied with an external power supply and are of the order of $0.01 \mathrm{~T}$. The substrates used for all the experiments are of standard glass $\left(38 \times 25 \times 1 \mathrm{~mm}^{3}\right)$. The substrates are cleaned with acetone in an ultrasonic bath for fifteen minutes and then treated with basic piranhas olution (ultra-pure water/ammonia/hydrogen peroxide with the ratio 5:3:1) at $67^{\circ} \mathrm{C}$ for forty minutes. After each cleaning stage, the substrates are rinsed with ultrapure water. The cleaned substrates are then dried by nitrogen blow. The aqueous superparamagnetic colloids (polystyrene coated magnetite) are obtained from Polysciences, Inc. at a concentration of $2.52 \%(\mathrm{w} / \mathrm{v})$. They are polydisperse with diameters ranging from 1 to $2 \mu \mathrm{m}$. The suspension (as provided) is ultrasonicated before commencing experiments. We investigate the coating process by pouring $120 \mu \mathrm{l}$ of that suspension on a spinning substrate. We perform the experiments with an applied magnetic field and at zero-field conditions. After the deposit is completely dried, micrographies are taken at $2 \mathrm{~mm}$ intervals from the center of rotation. The images are thresholded, segmented and then analyzed through home made routines in Octave ${ }^{\circledR}$.

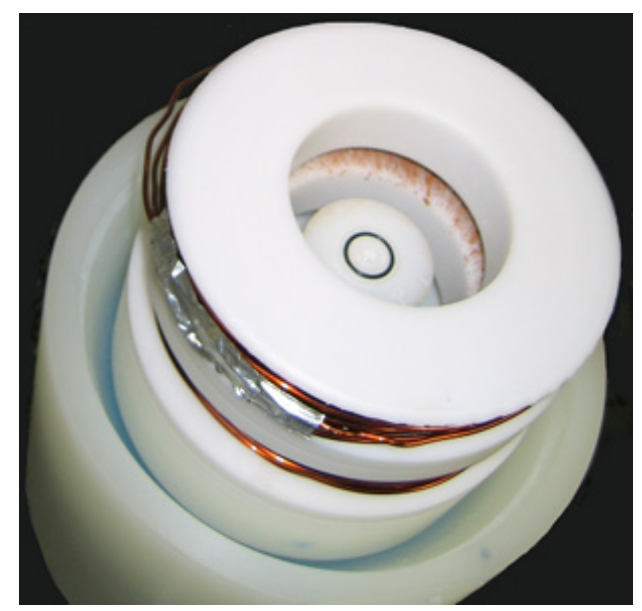

Fig. 1. Experimental configuration of the spin-coater with Helmholtz coils. 
Spin-coating of dilute magnetic colloids in a magnetic field

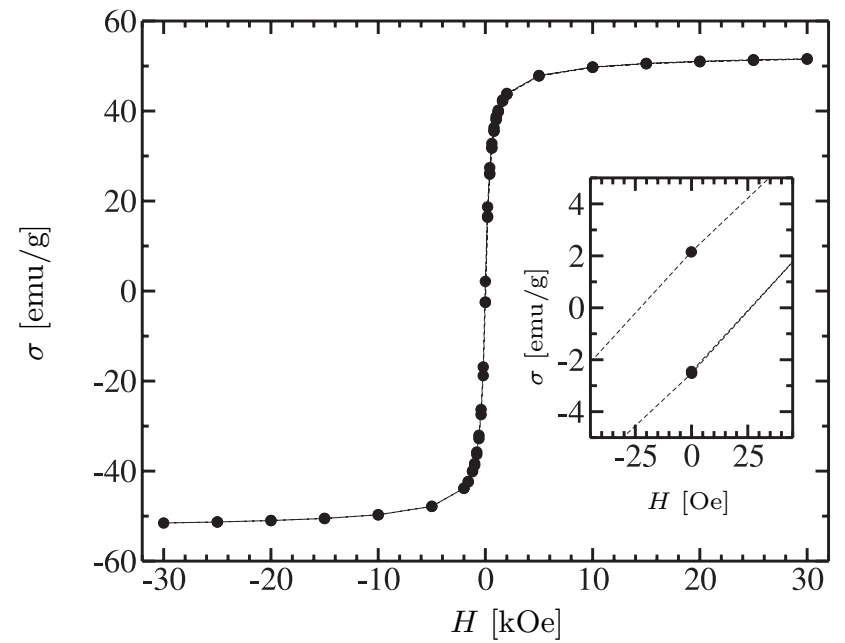

Fig. 2. Magnetization curve for the magnetic particles at $300 \mathrm{~K}$. Inset shows a magnified portion of the graph for obtaining the coercive field strength.

Magnetic characterization of the colloidal particles is done with a SQUID magnetometer (Lot-Oriel n. MPMS-XL). In Fig. 2, the magnetization curve is shown, with a small coercive field strength $\left(H_{c}=23 \mathrm{Oe}\right.$; see inset). Also, the mass magnetization at a moderate field $(1.2 \mathrm{kOe})$ is about $40 \mathrm{emu} / \mathrm{g}$. This indicates that the particles are superparamagnetic. The volume magnetic susceptibility at $H=0$ is 1.24 .

2. Results and discussion. In our experimental conditions, the deposits of colloidal particles are sub-monolayers, mainly formed by clusters of particles. The microscopic image of the dried substrate at an applied field of $0.033 \mathrm{~T}$ and a rotation rate of $2000 \mathrm{rpm}$ is shown in Fig. 3, right. To compare this condition, we include a corresponding image at zero-field (Fig. 3, left). Both images are qualitatively representative for the field and zero-field conditions, respectively. Other experimental parameters are unchanged. In general, we obtain less dense sub-monolayer morphologies for different conditions: (i) if we move away from the center of rotation on the dried substrate; (ii) if the rotation rate is increased; (iii) if the applied magnetic field is decreased.

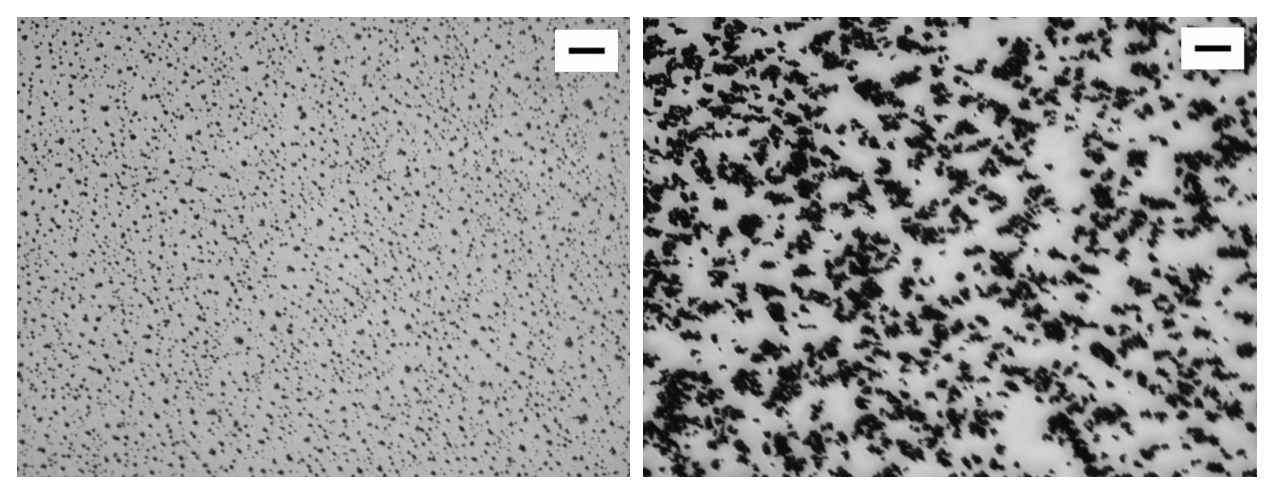

Fig. 3. Micrographies of the dried substrate at $4 \mathrm{~mm}$ from the center. During the spinning $(2000 \mathrm{rpm})$, the applied field was $0 \mathrm{~T}$ (left) and $0.033 \mathrm{~T}$ (right). Scale bars are $0.1 \mathrm{~mm}$. 

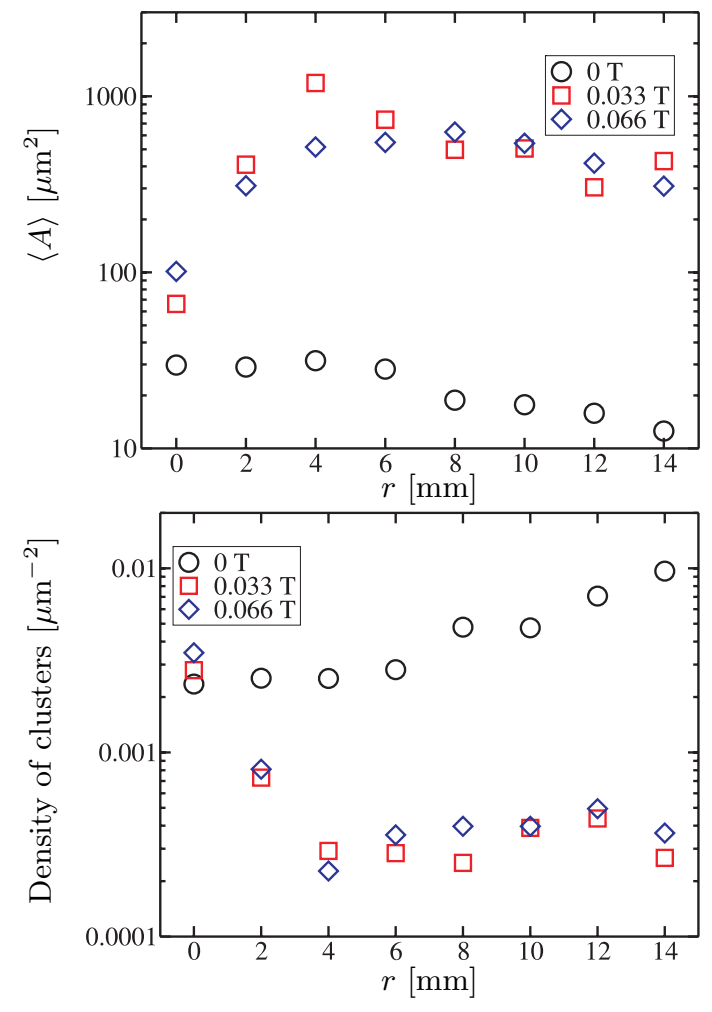

Fig. 4. Average area (top) and number density (bottom) of clusters as a function of distance from the center of rotation $r$ at $2000 \mathrm{rpm}$.

Fig. 4 (top) shows the average area $\langle A\rangle$ (in fact, the area of projection on the dried substrate) of clusters at increasing distances from the center of rotation $(r)$. It can be seen that the average area of clusters is higher (squares and diamonds) than for the case of zero-field condition (circles). We measured the density of clusters (number density) for the above-mentioned distances (Fig. 4, bottom). The variation in values represents the non-planarization phenomenon in spin-coating $[7]$.

For all the properties, which we measure in the experiment (e.g., mean area), we extract the mean and standard deviation of the values corresponding to different distances to the center $r$, and plot them against the applied magnetic field (e.g., for the mean area, see Fig. 5, top). In the latter plot, the bars, which correspond to the standard deviation mentioned above, are longer with the field compared to the zero-field case (take into account the log scale). This occurs due to a larger dependence of $\langle A\rangle$ on $r$. Altogether, the mean area increases as we increase the applied field. The number density of clusters (Fig. 5, bottom) decreases as the applied field is increased. Also, we can see from the plot that the dependence on $\omega$ (rotation rate) and on $r$ becomes weaker.

The morphological transition (from sparse to sub-monolayer deposits) are commonly observed in our experiments, where the magnetic field is applied. The applied field influences the transition mainly through the following phenomena. Firstly, it changes the dynamic viscosity of the magnetic colloidal dispersion spinning on the substrate. This has an impact on the rheology of the dispersion. Secondly, the field acts on the particles, which controls the clustering behaviour 

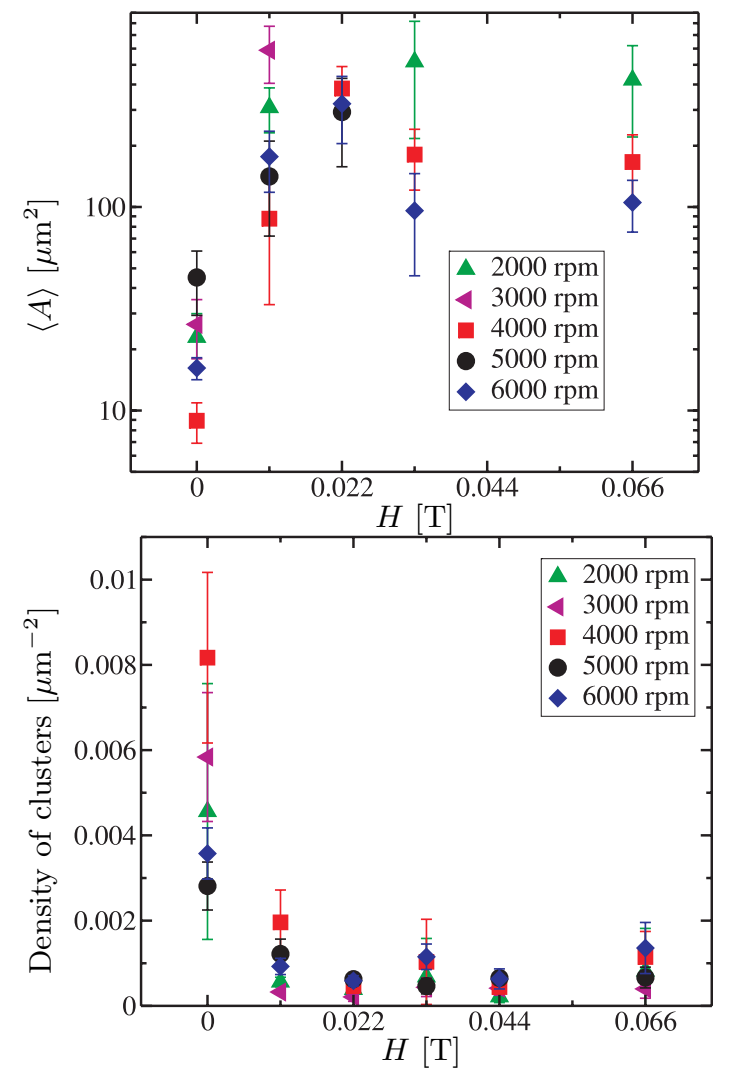

Fig. 5. Mean area (top) and mean number density (bottom) of clusters as a function of an external magnetic field.

through inter-particle dipole interactions. The increasing applied field enhances the interaction between particles, which drives to link the neighboring clusters together to form sub-monolayer deposits (increasing mean area in Fig. 5, top, and decreasing the mean number density in Fig. 5, bottom).

But in the case of zero-field, the colloidal dispersion behaves as non-magnetic (particles are superparamagnetic, with a very small remanent magnetization). Consequently, the morphology of the coating is determined by the rotation rate and static parameters like the initial concentration (constant in our experiments) and solvent properties. Compared to other simpler systems, the feature of having a polydisperse dispersion has a relevant effect, which will be subjected to further studies.

The local occupation factor is defined as the area occupied by the clusters relative to the total area of the region. This property is equivalent to the thickness in a multi-layer coating. For the given experimental condition, it depends on $r$ (non-planarization). Globally, on increasing the applied field, the increase in mean occupation factor (Fig. 6) could be understood by means of an increase of the effective viscosity, which makes the evaporation of the solvent more relevant during the coating. At higher fields, the ocupation factor seems to saturate.

In the case of high initial concentrations, the spin-coating of non-magnetic colloids leads to different symmetries (e.g., four-fold vs. six-fold) depending on the dispersion parameters and rotation rate $[6,7]$. For the case of magnetic colloids, 


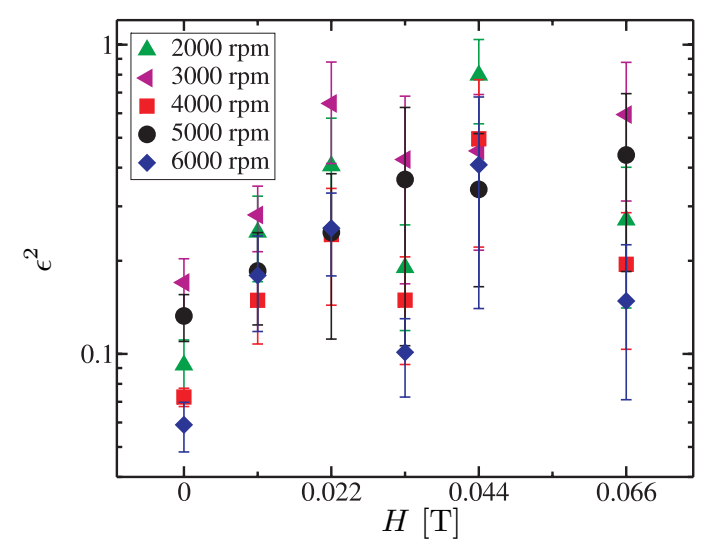

Fig. 6. Mean occupation factor of clusters as a function of an external magnetic field.
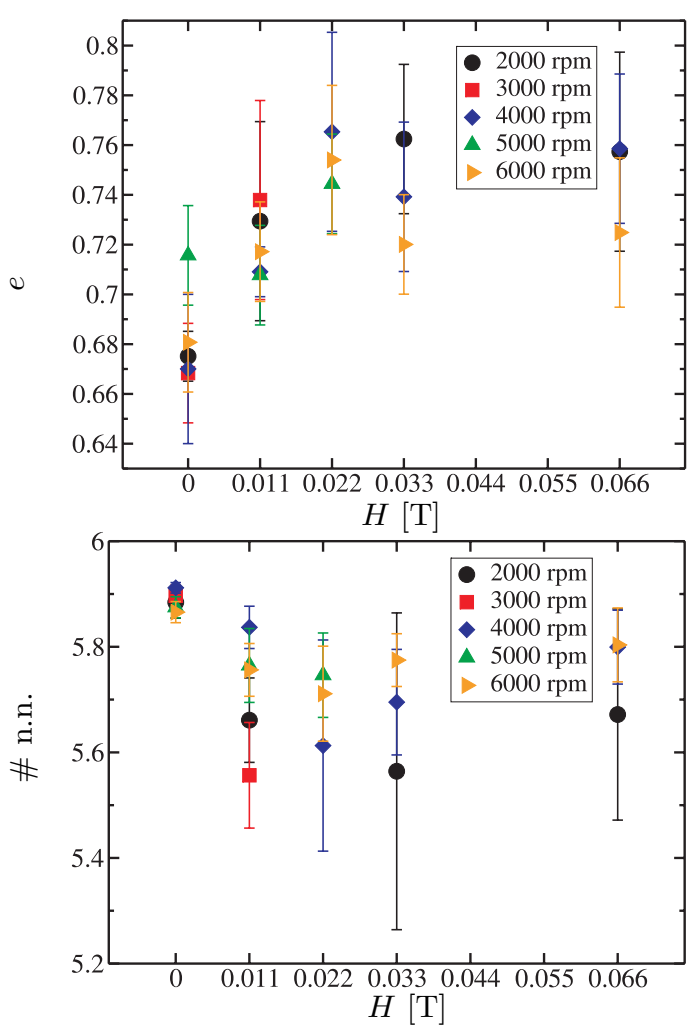

Fig. 7. Mean eccentricity (top) and mean number of nearest neighbours (bottom) of clusters as a function of an external magnetic field.

the applied field or the rotation may create some preferred direction that could influence the order in the coating, or the corresponding thresholds. To study this phenomenon in our dilute dispersions, we characterize the geometry and the orientation of the clusters. First, we measure the eccentricity of a cluster by means of the second order central moment. The mean eccentricity of the clusters increases with increasing the applied field strength (Fig. 7, top). The eccentricity is a positive defined number, consequently, even at $H=0$ the eccentricity is not 


\section{Spin-coating of dilute magnetic colloids in a magnetic field}

zero. We found that within the experimental resolution, the clusters were not oriented (data not shown). So, there is no privileged direction in our experimental conditions.

Finally, we analyze the mean number of nearest neighbors of each cluster. We find the centroid positions for each cluster and calculate the corresponding Voronoi diagram [24]. We observe (Fig. 7, bottom) that in the zero-field case, the value is close to six and with very low dispersion in data. When we apply the field, their values decrease, with an increased error bar. In a hexagonal lattice, the most common defect is the penta-hepta, which will not lead to a decrease of the mean value of nearest neighbours. Thus, this result is of another nature and it could be that the organization of clusters tends to be more disordered and with more fraction of the square lattice. Actually, this is what is observed in concentrated dispersions at zero field when the viscosity is increased $[6,7]$.

3. Conclusion. Hydrodynamic interactions are dominant in aqueous-based superparamagnetic colloids at zero-field condition. Applied magnetic fields increase the effective viscosity and change the aggregation dynamics of the particles. Morphological transition from sparse to sub-monolayer deposits occurs, which increases the mean area and decreases the mean number density of clusters. The dependence of the occupation factor on the distance from the center of rotation confirms the non-planarization in spin-coating processes. On the one hand, we conclude that under these experimental conditions, the external field together with the magnetic dipole interactions is not enough to define a preferred direction, but only to increase the characteristic time through effective viscosity. A slower hydrodynamics allows a longer interaction among particles and a higher effect of evaporation. This means a higher occupation factor and more eccentric clusters. On the other hand, we observe that most of the properties saturate when the magnetic field is further increased. Ordering mechanisms result mostly from the hydrodynamics, and the main effect of the magnetic field is to change the rheology. Further experiments with monodisperse suspensions will complement this results. Also, the mechanisms involved in the transition from sub-monolayer to compact structures will be considered.

Acknowledgements. We acknowledge C. Gómez-Polo for her generous loan of SQUID, and to J.M. Pastor and M.A. Miranda for the magnetic characterization of the colloidal particles (Fig. 2). This work is partly supported by the Spanish Government (contract No. FIS2008-01126) and by the Gobierno de Navarra (Departamento de Educación). MP acknowledges the financial support from the "Asociación de Amigos de la Universidad de Navarra".

\section{REFERENCES}

[1] J.-S. Gau. Magnetic thin film devices. Mat. Sci. Eng. B, vol. 3 (1989), pp. $377-381$.

[2] D. WeBb. Microwave magnetic thin-film devices. IEEE T. Magn., vol. 24 (1988), pp. 2799-2804.

[3] N. Shukla And M. Nigra. Synthesis and self-assembly of magnetic nanoparticles. Surf. Sci., vol. 601 (2007), pp. 2615-2617.

[4] M. Golosovtsky, Y.SaAdo, And D. Davidov. Self-assembly of floating magnetic particles into ordered structures: A promising route for the fabrica- 


\section{Pichumani, W. González-Viñas}

tion of tunable photonic band gap materials. Appl. Phys. Lett., vol. 75 (1999), pp. $4168-4170$.

[5] A. Mıнi, M. OcaÑA, AND H. Míguez. Oriented colloidal-crystal thin films by spin-coating microspheres dispersed in volatile media. Adv. Mater., vol. 18 (2006), pp. 2244-2249.

[6] C. Arcos, et al. Orientationally correlated colloidal polycrystals without long-range positional order. Phys. Rev. E, vol. 77 (2008), p. 050402(R).

[7] M. Giuliani, W. González-Viñas, K. Poduska, and A. Yethiraj. Dynamics of crystal structure formation in spincoated colloidal films. J. Phys. Chem. Lett., vol. 1 (2010), pp. 1481-1486.

[8] J. Weis And D. Levesque. Chain formation in low density dipolar hard spheres: A monte carlo studyj. Phys. Rev. Lett., vol. 71 (1993), pp. 27292732 .

[9] P. Camp and G. Patey. Structure and scattering in colloidal ferrofluids. Phys. Rev. E, vol. 62 (2000), pp. 5403-5408.

[10] T. Kruse, A. Spanoudaki, and R. Pelster. Monte Carlo simulations of polydisperse ferrofluids: Cluster formation and field-dependent microstructure. Phys. Rev. B, vol. 68 (2003), p. 054208.

[11] Z. WANG AND C. Holm. Structure and magnetic properties of polydisperse ferrofluids: A molecular dynamics study. Phys. Rev. E, vol. 68 (2003), p. 041401.

[12] J. Huang And C. Holm. Magnetization of polydisperse colloidal ferrofluids: Effect of magnetostriction. Phys. Rev. E, vol. 70 (2004), p. 061404.

[13] C. Holm, et al. Equilibrium properties of a bidisperse ferrofluid with chain aggregates: theory and computer simulations. J. Phys.: Condens. Matter, vol. 18 (2006), pp. S2737-S2756.

[14] G. Ganzenmüller and P. CAmp. Vapor-liquid coexistence in fluids of charged hard dumbbells. J. Chem. Phys., vol. 126 (2007), p. 191104.

[15] J. JoRDANOVIC AND S. KLAPP. Field-induced layer formation in dipolar nanofilms. Phys. Rev. Lett., vol. 101 (2008), p. 038302.

[16] P. Domínguez-García And M. Rubio. Three-dimensional morphology of field-induced chain-like aggregates of superparamagnetic microparticles. Colloids Surf., A, vol. 358 (2010), pp. 21-27.

[17] S. Melle, M. Rubio, And G. Fuller. Orientation dynamics of magnetorheological fluids subject to rotating external fields. Int. J. Mod. Phys. B, vol. 15 (2001), pp. 758-766.

[18] A.G. Emslie, F.T. Bonner, And L.G. PeCK. Flow of a viscous fluid on a rotating disk. J. Appl. Phys., vol. 29 (1958), pp. 858-862.

[19] D. Meyerhofer. Characteristics of resist films produced by spinning. J. Appl. Phys., vol. 49 (1978), no. 7, pp. 3993-3997.

[20] D.P. Birnie III AND M. Manley. Combined flow and evaporation of fluid on spinning disk. Phys. of Fluids, vol. 9 (1997), no. 4, pp. 870-875. 
[21] T. Rehg And B. Higgins. Spin coating of colloidal suspensions. AIChe J., vol. 38 (1992), pp. 489-501.

[22] L. Shereda, R. Larson, and M. Solomon. Local stress control of spatiotemporal ordering of colloidal crystals in complex flows. Phys. Rev. Lett., vol. 101 (2008), p. 038301.

[23] B.J. Ackerson and N.A. Clark. Shear-induced melting. Phys. Rev. Lett., vol. 46 (1981), no. 2, pp. 123-126.

[24] G. Voronoï. Nouvelles applications des paramètres continus à théorie des forms quadratiques. Deuxième Mémoire: Recherches sur les parallelodères primitifs. J. reine angew. Math., vol. 134 (1908), pp. 198-287.

Received 16.06.2011 